\title{
VVV WIN 1733-3349: a low extinction window to probe the far side of the Milky Way bulge
}

\author{
R. K. Saito ${ }^{1} \dagger$, D. Minniti ${ }^{2,3,4}$, R. A. Benjamin ${ }^{5}$, M. G. Navarro $2,6,3$, \\ J. Alonso-García 7,3 , O. A. Gonzalez ${ }^{8}$, R. Kammers ${ }^{1}$, F. Surot ${ }^{9}$ \\ ${ }^{1}$ Departamento de Física, Universidade Federal de Santa Catarina, Trindade 88040-900, Florianópolis, SC, Brazil \\ ${ }^{2}$ Departamento de Fisica, Facultad de Ciencias Exactas, Universidad Andres Bello, Av. Fernandez Concha 700, Las Condes, \\ Santiago, Chile \\ ${ }^{3}$ Instituto Milenio de Astrofísica, Santiago, Chile \\ ${ }^{4}$ Vatican Observatory, V00120 Vatican City State, Italy \\ ${ }^{5}$ Department of Physics, University of Wisconsin-Whitewater, $800 \mathrm{~W}$ Main St, Whitewater, WI 53190 USA \\ 6 Dipartimento di Fisica, Università degli Studi di Roma "La Sapienza", P.le Aldo Moro, 2, I00185 Rome, Italy \\ ${ }^{7}$ Centro de Astronomía (CITEVA), Universidad de Antofagasta, Av. Angamos 601, Antofagasta, Chile \\ ${ }^{8}$ UK Astronomy Technology Centre, Royal Observatory, Blackford Hill, Edinburgh EH9 3HJ, UK \\ ${ }^{9}$ Instituto de Astrofísica de Canarias, E-38205, La Laguna, Tenerife, Spain
}

Accepted XXX. Received YYY; in original form ZZZ

\begin{abstract}
Windows of low extinction in the Milky Way (MW) have been used along the past decades for the study of the Galactic structure and the stellar population across the inner bulge and disk. Here we report the analysis of another low extinction nearIR window discovered by the VISTA Variables in the Vía Láctea Survey. VVV WIN 1733-3349 is about half a degree in size and is conveniently located right in the MW plane, at Galactic coordinates $(l, b)=(-5.2,-0.3)$. The mean extinction of VVV WIN 1733-3349 is $A_{K s}=0.61 \pm 0.08 \mathrm{mag}$, which is much smaller than the extinction in the surrounding area. The excess in the star counts is consistent with the reduced extinction, and complemented by studying the distribution of red clump (RC) stars. Thanks to the strategic low-latitude location of VVV WIN 1733-3349, we are able to interpret their RC density fluctuations with the expected overdensities due to the presence of the spiral arms beyond the bulge. In addition, we find a clear excess in the number of microlensing events within the window, which corroborates our interpretation that VVV WIN 1733-3349 is revealing the far side of the MW bulge.
\end{abstract}

Key words: Galaxy: bulge - Galaxy: structure - dust, extinction - Surveys Catalogues

\section{INTRODUCTION}

Windows of low extinction in the Milky Way are very important in Astronomy. A classical example, relevant to the study of star forming regions, is the low optical extinction window that allowed the deep exploration of the innermost part of the Orion Giant Molecular Cloud revealing the secrets of the Trapezium cluster and imaging the first protoplanetary

* Based on observations taken within the ESO Public Surveys VVV and VVVX, Programme IDs 179.B-2002 and 198.B-2004, respectively.

$\dagger$ E-mail: saito@astro.ufsc.br disks with the Hubble Space Telescope (HST; O'dell, \& Wen 1994; O'dell, \& Wong 1996). Another important example is Baade's window, an optical extinction window of roughly half a degree in size centred at Galactic coordinates $(l, b)=(1,-4) \mathrm{deg}$, that allowed the study of the bulge stellar populations (Baade 1946; Baade, \& Gaposchkin 1963; Blanco, \& Blanco 1985) and it continues to be used as a reference field due to its detailed characterisation (see Barbuy et al. 2018 for a review).

Some other well known examples of low extinction windows used to study Galactic structure and stellar populations are: Sgr windows (Baade, \& Gaposchkin 1963; Lloyd Evans 1976), Plaut's window (Plaut 1970; Oort, \& Plaut 
1975; Glass, \& Feast 1982), Sweeps window (Stanek 1998; Clarkson et al. 2008), W0.2-2.1 and W359.4-3.1 windows (Dutra et al. 2002), Centaurus window (Bica et al. 2005), etc.

Much of what we initially learned about the bulge populations was based on the study of such low extinction windows that allowed us to see deep into the Galactic bulge (e.g., Lloyd Evans 1976; Whitford 1978; Blanco et al. 1984; Terndrup 1988; Stanek 1996; Frogel et al. 1999) and also to constrain the star count models of the Milky Way like the Bensançon model (e.g., Robin, \& Creze 1986). Recent studies of these bulge windows carried out with the HST have allowed to explore the complex star formation history (e.g., Clarkson et al. 2011; Haywood et al. 2016; Bernard et al. 2018).

The advent of large IR surveys such as the Two Micron All Sky Survey (2MASS; Skrutskie et al. 2006), the VISTA Variables in the Vía Láctea Survey (VVV; Minniti et al. 2010) in the near-IR, the Spitzer Galactic Legacy Infrared Mid-Plane Survey Extraordinaire (GLIMPSE; Benjamin et al. 2003), and the Wide-field Infrared Survey Explorer (WISE; Wright et al. 2010) in the mid-IR, enabled to make detailed reddening and extinction maps, to obtain a more global view of inner Galactic structure and stellar populations, and also to search for additional windows of low extinction located at low Galactic latitudes.

Our VVV Survey in particular has recently produced a variety of extinction maps towards the Galactic bulge (Gonzalez et al. 2011, 2018; Schultheis et al. 2014; Minniti et al. 2014, 2018; Alonso-García et al. 2018; Soto et al. 2013, 2019). Using these near-IR extinction maps we have recently reported the discovery of VVV WIN 1713-3939 (a.k.a. Dante's window $)$, located at $(l, b)=(347.4,-0.4) \mathrm{deg}$, that allowed the identification of the spiral arm structure in the far side of the Milky Way (Minniti et al. 2018).

In this paper we present the characterisation of another low extinction near-IR window of roughly half a degree in size, located in the Galactic plane at Galactic coordinates $(l, b)=(-5.2,-0.3) \mathrm{deg}$, that exhibits not only an overdensity in the star counts, but also a clear excess of microlensing events with respect to its surrounding regions.

\section{OBSERVATIONS}

The ESO VISTA Variables in Vía Láctea (VVV) survey has recently completed near-IR observations of $562 \mathrm{sq}$. deg. area of the MW bulge and the adjacent plane. The VVV strategy consisted in two sets of quasi simultaneous $Z Y$ and $J H K_{\mathrm{S}}$ observations, plus 50-100 observations in $K_{\mathrm{S}}$ band over several years $(2010-2015)$, providing a deep, high-resolution dataset of the inner Galaxy in the near-IR (Minniti et al. 2010; Saito et al. 2012).

The standard VVV photometry is based on aperture photometry on the stacked VVV tile images, and provided by the Cambridge Astronomical Survey Unit (CASU; for details see Saito et al. 2012). More recently, point-spread function (PSF) photometry was performed on the VVV images, and catalogues with data in the different VVV filters have been released for the inner regions of our Galaxy (e.g., Alonso-García et al. 2018; Surot et al. 2019). Due to a high

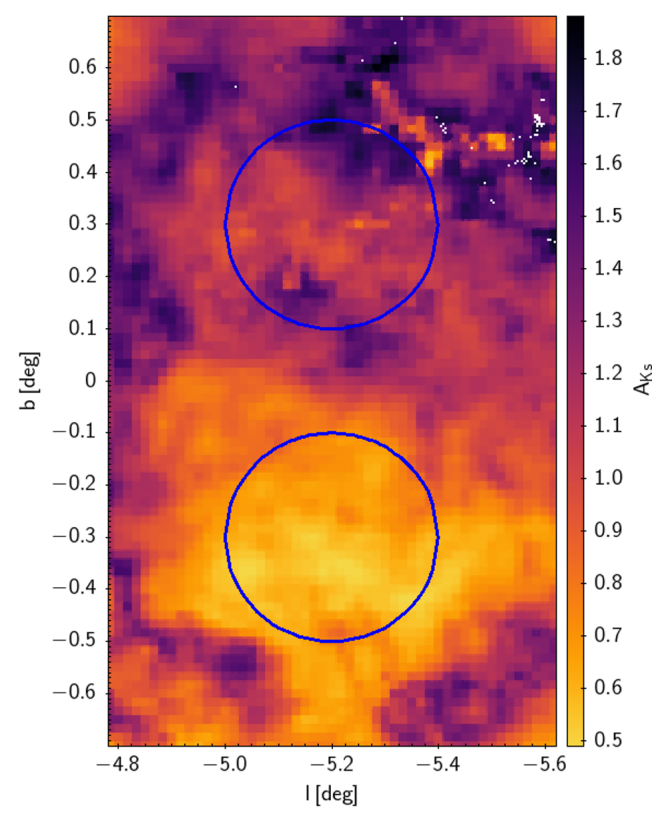

Figure 1. A modified version of the VVV extinction map of Surot et al. (2019) for the region around VVV WIN 1733-3349. Two circles mark the regions defined as "window" (at negative latitudes) and "control field" (positive $b$ ).

crowding in the inner bulge - where the window is located the VVV data presented here are based on PSF photometry.

\section{DISCUSSION}

Gonzalez et al. (2018) made use of VVV red clump stars detected in Alonso-García et al. (2018) catalogues to create a high-resolution reddening map of the inner region of the Milky Way bulge $(-10<l<10 \mathrm{deg}$ and $-1.5<b<1.5 \mathrm{deg})$. They discovered the presence of a low extinction window right in the Galactic plane $(b \sim-0.3 \mathrm{deg})$, at just about five degrees from the Galactic centre $(l \sim-5.2 \mathrm{deg})$. This window named VVV WIN 1733-3349 in Gonzalez et al. (2018) is also clearly seen in the maps of Surot et al. (2019) and in the distribution of Gaia DR2 sources (Gaia Collaboration et al. 2018). The region is about half degree in size, as can be appreciated in Fig. 1, which shows an adapted version of the Surot et al. (2019) map for the region around the window.

In our analysis we defined as the window a circular region of $24^{\prime}$ diameter centered at $(l, b)=(-5.2,-0.3)$ deg, corresponding to RA, Dec $(\mathrm{J} 2000)=17: 33: 51.92,-33: 29: 56.92$ in the equatorial system. The position of WIN 1733-3349 (a.k.a. Oscar's window) is especially important since for a latitude of $b=-0.3 \mathrm{deg}$ the vertical projection along the line of sight is small $(z<100 \mathrm{pc})$ even at the far side of the MW $\operatorname{disk}(d \lesssim 18 \mathrm{kpc})$.

\subsection{Star counts and colour-magnitude diagrams}

Using the Nishiyama et al. (2009) extinction law, we measured $A_{K s}=0.61 \pm 0.08 \mathrm{mag}$ for VVV WIN 1733-3349 from the Surot et al. (2019) extinction maps, which is much smaller than the extinction in the surrounding area. For instance, for the symmetric area at positive latitude $\left(24^{\prime}\right.$ di- 

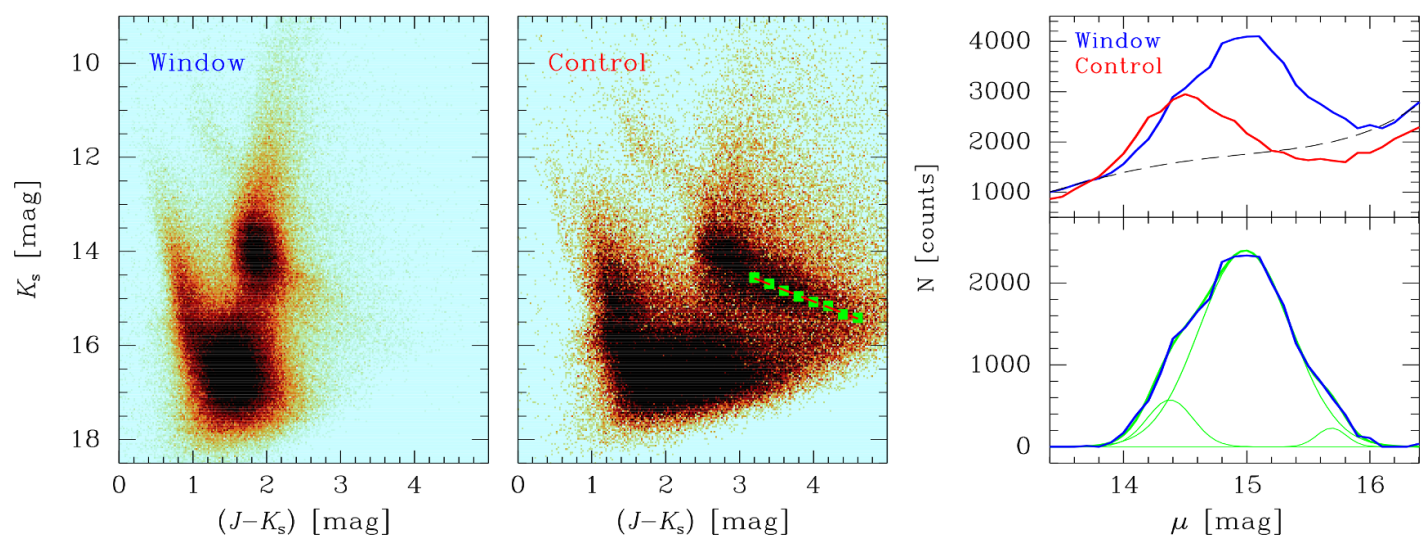

Figure 2. $K_{\mathrm{S}}$ vs $\left(J-K_{\mathrm{S}}\right)$ colour magnitude diagram (CMD) for the window (left-panel) and control field (central-panel). In the CMD for the control field a dashed line mark the slope of $A_{K s}=(0.612 \pm 0.018) \times E\left(J-K_{\mathrm{S}}\right)$ measured for the reddening vector. Top-right panel: distribution of selected RC versus distance moduli of VVV WIN 1733-3349 (blue line) and of the control field (red line). The polynomial fit used to subtract the background LF of VVV WIN 1733-3349 is also shown with a dashed line. Bottom-right panel: multi Gaussian fit for the distribution of window RC stars after subtracting a polynomial fit to the luminosity function. Information about the three peaks are listed in Table 1.

ameter area centered at $l, b=-5.2,+0.3$ deg.) the extinction is roughly twice as in the window, with $A_{K s}=1.20 \pm 018 \mathrm{mag}$. This symmetric area at positive latitude can be used as a control field because there is negligible disk warping at these coordinates (e.g., Momany et al. 2006). Considering similar stellar populations in both regions above and below the plane (window and control field, respectively), the differences observed in comparing them can be interpreted as caused by different extinction levels.

Fig. 2 shows the $K_{\mathrm{S}} v s\left(J-K_{\mathrm{S}}\right)$ colour magnitude diagram (CMD) for VVV WIN 1733-3349 (left-panel) and the control field (right-panel). The difference in the extinction level is remarkable, with the CMD for the control field stretched along the reddening vector. While producing the CMDs, an examination on the stellar density shows a higher density of sources within WIN 1733-3349 in all VVV filters when compared to the control field. Indeed, the stellar density in the window is about two times larger than that of the control field. The difference in the stellar density is even larger in the optical Gaia DR2 data (Gaia Collaboration et al. 2018), with about four times more stars within the window compared with the control field. Similar ratio is also seen in the optical Pan-STARRS1 (Chambers et al. 2016) and DECaPS data (Schlafly et al. 2018).

Fig. 3 presents in the mid panel a density map for the optical Gaia DR2 data. The spatial distribution shows an overdensity in agreement with the location and size of the of WIN 1733-3349 as seen in the extinction map (see Fig. 1). The distribution in distance for the Gaia DR2 sources within WIN 1733-3349 compared with the control field (from Bailer-Jones et al. 2018, bottom panel of Fig. 3) shows that the Gaia photometry is not deep enough, reaching barely to the bulge stars $(\sim 7 \mathrm{kpc})$.

We can explore zones with more transparency towards the inner MW bulge in order to map structures towards - or even behind - the bulge. For this we made use of red clump stars as distance indicators within VVV WIN 1733-3349. We selected red clump stars using a simple cut in colour in the CMDs and then calculated the distance assuming
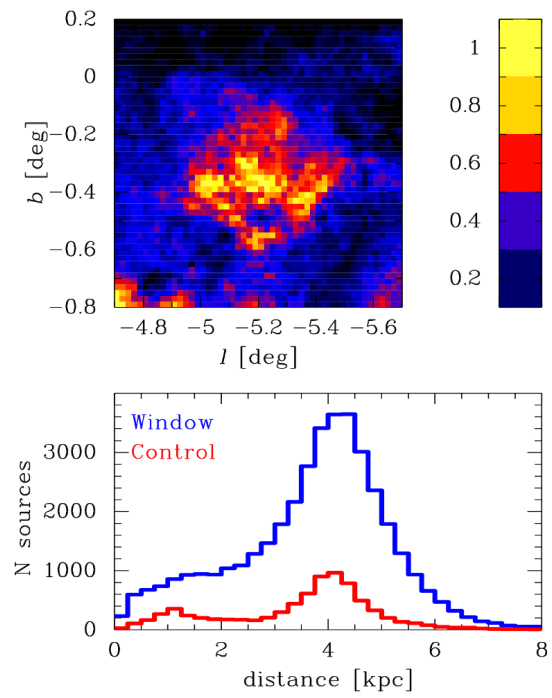

Figure 3. Top panel: density map of Gaia DR2 ( $G$ band) sources around the window normalized by the maximum density value. A vertical bar shows the colour code in the map. Bottom panel: distribution in distance for the Gaia DR2 sources according to Bailer-Jones et al. (2018).

the recently calibrated absolute magnitude of RC stars provided by Gaia DR2 as $M_{K s}=-1.605 \pm 0.009$ and $\left(J-K_{\mathrm{S}}\right)_{0}=$ $0.66 \pm 0.02 \mathrm{mag}$ (Ruiz-Dern et al. 2018) and the slope of the reddening vector measured directly from the control field CMD which yields to $A_{K s}=(0.612 \pm 0.018) \times E\left(J-K_{\mathrm{S}}\right)$. With those values the distance modulus for the RC stars is given by $\mu=-5+5 \log d(p c)=K_{\mathrm{S}}-0.612 \times\left(J-K_{\mathrm{S}}\right)+$ $2.009 \mathrm{mag}$. The slope of the reddening vector is flatter than $A_{K s}=0.725 \times E\left(J-K_{\mathrm{S}}\right)$ from Schlegel et al. (1998), but steeper than $A_{K s}=0.528 \times E\left(J-K_{\mathrm{S}}\right)$ from Nishiyama et al. (2009). These values are much steeper than the slope in Alonso-García et al. (2017) and Minniti et al. (2018), where $A_{K s}=0.428 \times E\left(J-K_{\mathrm{S}}\right)$ and $A_{K s}=0.484 \times E\left(J-K_{\mathrm{S}}\right)$, re- 


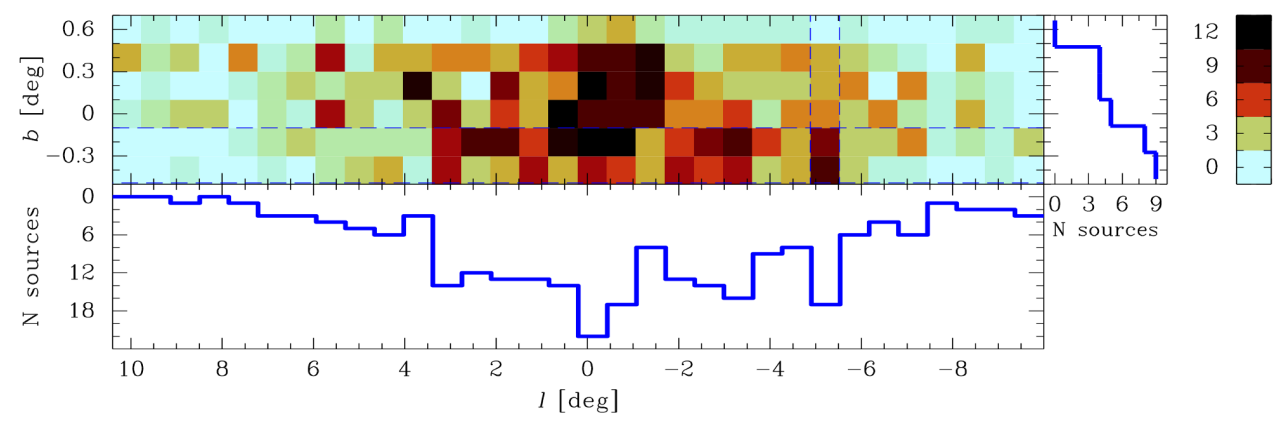

Figure 4. Density plot with the distribution of microlensing events in the inner bulge. An overdensity of events is present at the position of VVV WIN 1733-3349 ( $l, b=-5.2,-0.3 \mathrm{deg}$ ). Histograms for sources within the WIN 1733-3349 limiting coordinates (dashed lines) are also shown for both axes. A vertical bar shows the colour code in the map. Adapted from Navarro et al. (2018, 2019).

spectively. The use of a different extinction law causes the distance scale to shrink/expand.

The luminosity function (LF) for the WIN 1733-3349 RC stars compared with the control field RC stars is shown in the top-right panel of Fig. 2. The distributions are quite different, with many more RC stars in the window LF, which peaks much farther when compared with the control field and presents a composite distribution. In order to subtract the background and enhance possible peaks of the RC along the line of sight, In order to subtract the background and enhance possible peaks of the $\mathrm{RC}$ along the line of sight, we applied a polynomial + multi Gaussian fit, as shown in the bottom-right panel of Fig. 2. The resulting peaks due to the different $\mathrm{RC}$ are fitted with a Levenberg-Marquardt optimization algorithm (e.g., Kanzow et al. 2004) in order to obtain their mean distances and respective standard deviations, which are listed in Table 1.

At the magnitude range of the fainter RC stars the completeness of the VVV PSF data on Galactic plane is over $80 \%$ (e.g., Saito et al. 2012; Surot et al. 2019). Following the discussion presented in Gonzalez et al. (2018), we assume that secondary peaks in the $\mathrm{RC}$ distributions are not caused by the red giant branch bump (e.g., Nataf et al. 2011, 2013). In contrast with the distances from Gaia DR2 as estimated by Bailer-Jones et al. (2018), the distances to the $\mathrm{RC}$ giants measured from the VVV near-IR photometry clearly penetrates through the Galaxy, out to $\sim 14 \mathrm{kpc}$. Using a flatter reddening vector in our calculations scales the $\mathrm{RC}$ distribution even to larger distances. For instance, with $A_{K s}=0.428 \times E\left(J-K_{\mathrm{S}}\right)$ from Alonso-García et al. (2017) the distances to the RC are $\sim 15 \%$ larger. This difference in slopes with previous VVV measurement could be due to the small number of RC stars at a given color in our control field, which, along with the relative closeness of the RC of the bulge and of the background spiral arm at these longitudes (Minniti et al, in prep.), complicates its accurate measurement.

\subsection{Microlensing}

Navarro et al. $(2018,2019)$ searched for microlensing events in bulge fields along the Galactic plane. They discovered several hundreds of events in a large area covering about 21 sq. deg. (within $-10.00<l<+10.44 \mathrm{deg}$, and $0.46<$ $b<+0.65 \mathrm{deg})$. The analysis of the spatial distribution of these microlensing events revealed an unexpected excess in

\begin{tabular}{ccl}
\hline \hline RC peaks & RC peaks & Interpretation \\
$\boldsymbol{\mu}\left(\boldsymbol{K}_{\mathrm{S}}\right)[\mathrm{mag}]$ & Dist. $[\mathrm{kpc}]$ & \\
\hline $14.376 \pm 0.373(\sim 13.51)$ & 7.50 & Scutum-Centaurus arm \\
$14.988 \pm 0.742(\sim 14.13)$ & 9.94 & Galactic bar \\
$15.689 \pm 0.236(\sim 14.85)$ & 13.73 & Sagittarius arm \\
\hline \hline
\end{tabular}

Table 1. Distances for the RC peaks from the multi Gaussian + polynomial fit to the window RC luminosity function.

the surface density of events at $(l, b)=(-5.2,-0.3) \mathrm{deg}$, in an area that corresponds to the location of the present window (see Fig. 4). This excess in the number density of events above the neighboring area is a 3 sigma result.

The traditional microlensing configurations (bulgebulge, bulge-disk and disk-disk events) should produce an excess of events in the very center of the MW due to the higher stellar density (Navarro et al. 2017), but not in this area. The most straightforward explanation is that we are seeing all the way through the bulge, including additional events that have lenses in the bulge and sources in the far disk. The first event with this new far disk-bulge event configuration was discovered by the United Kingdom Infrared Telescope Survey (UKIRT) at $(l, b)=(-0.12,-0.33)$ (Shvartzvald et al. 2017, 2018). Another candidate for far disk event at very low latitude was reported by Bennett et al. (2018) at $(l, b)=(0.90,-1.97)$. A more comprehensive study seems to be confirmed by the larger estimated distances of the RC sources in this region (Navarro et al. 2020, in preparation). We take this excess number of microlensing events in this area as additional evidence supporting the conclusion that VVV WIN 1733-3349 is a is a window of low near-IR extinction piercing deep through the entire bulge region.

\section{CONCLUSIONS}

We present the further characterisation of another window of low extinction in the Galactic bulge, VVV WIN 1733-3349 (a.k.a. Oscar's window). This window is strategically located in the plane, at $(l, b)=(-5.2,-0.3) \mathrm{deg}$, and has a diameter of about $24^{\prime}$. The window is clearly seen in the VVV extinction maps for the region as well as in the distribution of Gaia DR2 sources.

We use the deep VVV PSF photometry to measure the 
distance distribution of RC giants along the line of sight. The $\mathrm{RC}$ giants can be seen out to $\sim 14 \mathrm{kpc}$, in the far disk well beyond the bulge. A multi Gaussian fit shows the presence of three peaks, interpreted as the Scutum-Centaurus arm in the foreground disk, the Galactic bar and the Sagittarius arm (that is seen wrapping around behind the Galactic centre), at the distance of $d=7.06,9.37$ and $12.92 \mathrm{kpc}$, respectively. The distance of the Galactic bar and the Sagittarius arm coincide with distances for RC stars measured by Gonzalez et al. (2018) for the region within $|b|<1.5 \mathrm{deg}$.

We also find a clear excess of microlensing events in this window from the sample of Navarro et al. (2018, 2019), that is probably due to additional events that have lenses in the bulge and sources in the far disk. That is in agreement with our interpretation of the $\mathrm{RC}$ distribution, revealing the far side of the Milky Way bulge.

\section{ACKNOWLEDGEMENTS}

We gratefully acknowledge the use of data from the ESO Public Survey program IDs 179.B-2002 and 198.B-2004 taken with the VISTA telescope, and data products from the Cambridge Astronomical Survey Unit (CASU). This publication makes use of data products from the Wide-field Infrared Survey Explorer, which is a joint project of the University of California, Los Angeles, and the Jet Propulsion Laboratory/California Institute of Technology, funded by the National Aeronautics and Space Administration. R.K.S. acknowledges support from $\mathrm{CNPq} /$ Brazil through projects 308968/2016-6 and 421687/2016-9. Support for the authors is provided by the BASAL CONICYT Center for Astrophysics and Associated Technologies (CATA) through grant AFB-170002, and the Ministry for the Economy, Development, and Tourism, Programa Iniciativa Científica Milenio through grant IC120009, awarded to the Millennium Institute of Astrophysics (MAS). D.M. acknowledges support from FONDECYT through project Regular \#1170121.

\section{REFERENCES}

Alonso-García, J., Minniti, D., Catelan, M., et al. 2017, ApJ, 849, L13

Alonso-García, J., Saito, R. K., Hempel, M., et al. 2018, A\&A, 619, A4

Baade, W. 1946, PASP, 58, 249

Baade, W., \& Gaposchkin, C. H. P. 1963, Evolution of stars and galaxies

Bailer-Jones, C. A. L., Rybizki, J., Fouesneau, M., et al. 2018, AJ, 156, 58

Barbuy, B., Chiappini, C., \& Gerhard, O. 2018, ARA\&A, 56, 223

Benjamin, R. A., Churchwell, E., Babler, B. L., et al. 2003, PASP, 115,953

Bennett, D., Udalski, A., Bond, I., et al. 2018, AJ, 156, 11

Bernard, E. J., Schultheis, M., Di Matteo, P., et al. 2018, MNRAS, 477, 3507

Bica, E., Bonatto, C., Santiago, B. X., et al. 2005, A\&A, 433, 931

Blanco, V. M., McCarthy, M. F., \& Blanco, B. M. 1984, AJ, 89, 636

Blanco, V. M., \& Blanco, B. M. 1985, Mem. Soc. Astron. Italiana, 56,15

Chambers, K. C., Magnier, E. A., Metcalfe, N., et al. 2016, arXiv e-prints, arXiv:1612.05560
Clarkson, W., Sahu, K., Anderson, J., et al. 2008, ApJ, 684, 1110

Clarkson, W. I., Sahu, K. C., Anderson, J., et al. 2011, ApJ, 735, 37

Dutra, C. M., Santiago, B. X., \& Bica, E. 2002, A\&A, 381, 219

Frogel, J. A., Tiede, G. P., \& Kuchinski, L. E. 1999, AJ, 117, 2296

Gaia Collaboration, Brown, A. G. A., Vallenari, A., et al. 2018, A\&A, 616, A1

Glass, I. S., \& Feast, M. W. 1982, MNRAS, 198, 199

Gonzalez, O. A., Rejkuba, M., Zoccali, M., et al. 2011, A\&A, 534, A3

Gonzalez, O. A., Minniti, D., Valenti, E., et al. 2018, MNRAS, 481, L130

Haywood, M., Di Matteo, P., Snaith, O., et al. 2016, A\&A, 593, A82

Kanzow, C., Yamashita, N., Fukushima. M. 2004, JCam, 172, 375

Lloyd Evans, T. 1976, MNRAS, 174, 169

Minniti, D., Lucas, P. W., Emerson, J. P., et al. 2010, New Astron., 15, 433

Minniti, D., Saito, R. K., Gonzalez, O. A., et al. 2014, A\&A, 571, A91

Minniti, D., Saito, R. K., Gonzalez, O. A., et al. 2018, A\&A, 616, A26

Momany, Y., Zaggia, S., Gilmore, G., et al. 2006, A\&A, 451, 515

Nataf, D. M., Udalski, A., Gould, A., et al. 2011, ApJ, 730, 118

Nataf, D. M., Gould, A. P., Pinsonneault, M. H., et al. 2013, ApJ, 766,77

Navarro, M. G., Minniti, D. \& Contreras Ramos, R. 2017, ApJ, 851, L13

Navarro, M. G., Minniti, D., \& Contreras-Ramos, R. 2018, ApJ, 865, L5

Navarro, M. G., Contreras Ramos, R., Minniti, D., et al. 2019, arXiv e-prints, arXiv:1907.04339

Nishiyama, S., Tamura, M., Hatano, H., et al. 2009, ApJ, 696, 1407

O'dell, C. R., \& Wen, Z. 1994, ApJ, 436, 194

O'dell, C. R., \& Wong, K. 1996, AJ, 111, 846

Oort, J. H., \& Plaut, L. 1975, A\&A, 41, 71

Plaut, L. 1970, A\&A, 8, 341

Robin, A., \& Creze, M. 1986, A\&A, 157, 71

Ruiz-Dern, L., Babusiaux, C., Arenou, F., Turon, C., \& Lallement, R. 2018, A\&A, 609, A116

Saito, R. K., Hempel, M., Minniti, D., et al. 2012, A\&A, 537, A107

Schlegel, D. J., Finkbeiner, D. P., \& Davis, M. 1998, ApJ, 500, 525

Schlafly, E. F., Green, G. M., Lang, D., et al. 2018, ApJS, 234, 39

Schultheis, M., Zasowski, G., Allende Prieto, C., et al. 2014, AJ, 148,24

Shvartzvald, Y., Bryden, G., Gould, A., Henderson, C. B., Howell, S. B., \& Beichman, C., 2017, MNRAS, 457, 4089

Shvartzvald, Y., Novati, S. C., Gaudi, B. S., et al. 2018, ApJ, 857, L8

Skrutskie, M. F., Cutri, R. M., Stiening, R., et al. 2006, AJ, 131, 1163

Soto, M., Barbá, R., Gunthardt, G., et al. 2013, A\&A, 552, A101

Soto, M., Barbá, R., Minniti, D., et al. 2019, MNRAS, 488, 2650

Stanek, K. Z. 1996, ApJ, 460, L37

Stanek, K. Z. 1998, arXiv e-prints, astro-ph/9802307

Surot, F., Valenti, E., Hidalgo, S. L., et al. 2019, A\&A, 629, A1

Terndrup, D. M. 1988, AJ, 96, 884

Whitford, A. E. 1978, ApJ, 226, 777

Wright, E. L., Eisenhardt, P. R. M., Mainzer, A. K., et al. 2010 , AJ, 140,1868

This paper has been typeset from a $\mathrm{T}_{\mathrm{EX}} / \mathrm{IAT}_{\mathrm{EX}}$ file prepared by the author. 